SOUTH EAST EUROPE

Transnational Cooperation Programme
SNA P P 88 S E E

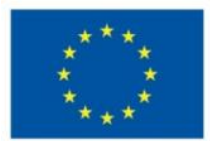

Programme co-funded by the EUROPEAN UNION

Stakeholder Konsultation bei der Anwendung Bester Praktiken zur nachhaltigen Baurohstoff-Planung

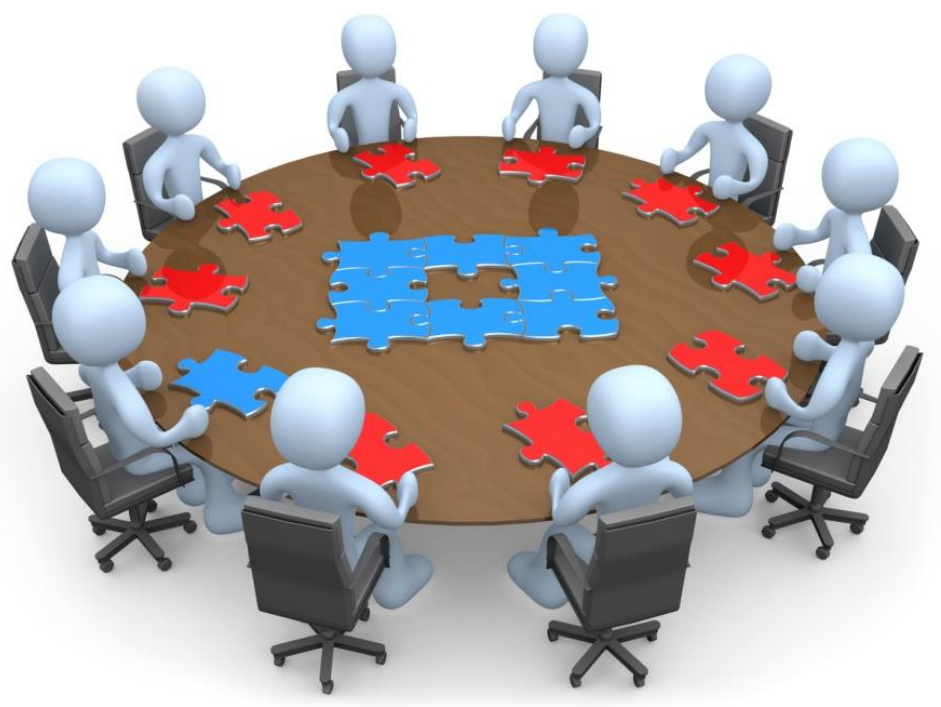

Juni 2014

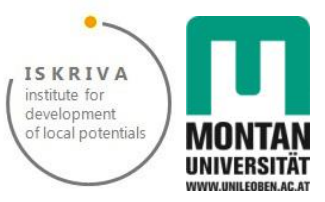




\section{Stakeholder Konsultation bei der Anwendung Bester Praktiken zur nachhaltigen Baurohstoff-Planung}

Basierend auf:

den Arbeiten und Erfahrungen der Projektpartner im Arbeitspaket 3 des SNAP-SEEProjekt " Baurohstoff-Planung in Süd-Ost Europa " (SEE/D/0167/2.4/X)

Ergebnis der Aktivität 3.3: Unterstützende Materialien für StakeholderKonsultationen: Handbuch über den Aufbau von Kapazitäten und StakeholderKonsultationen zur Baurohstoff Planung

Website: http://www.snapsee.eu

\section{Publication Information}

Editing \& Layout:

Cover Design:

Printing:

Year of Edition:
Foteini Stathogianni, Urška Dolinar

Stelios Mavrigiannakis

Geological Survey of Slovenia

2014

\section{Disclaimer}

Dieses Snap-SEE Veröffentlichung spiegelt nur die Meinung der Autoren. Die Verwaltungsbehörde des Programms Süd-Ost-Europa grenzüberschreitende Zusammenarbeit ist nicht verantwortlich für jegliche Verwendung der darin enthaltenen Informationen. (C) SNAP-SEE-Projekt 2014. 
Dolinar, Urška, MBA, Iskriva, institute for development of local potentials, Slovenia Softić, Mateja, Iskriva, institute for development of local potentials, Slovenia Kozinc, Zdravko, Iskriva, institute for development of local potentials, Slovenia

\section{Auftraggeber}

Montanuniversität Leoben (University of Leoben), Austria

\section{Rezensenten}

\begin{tabular}{ll}
\hline Name & Affiliation \\
\hline Shields, Deborah J., Dr. & University of Leoben, Austria \\
O'Brien, Jim & Former President of the European Aggregates Association, Ireland
\end{tabular}

\section{Acknowledgment}

Die Snap-SEE Projekt Partner danken der Europäischen Kommission für die Finanzierung dieses Projekts welches zu einer erfolgreichen Zusammenarbeit zwischen Partnern geführt hat, die ähnliche Probleme und eine gemeinsame Vision teilen, und damit die Entwicklung dieser Publication ermöglicht hat.

\section{Digital Object Identifier (DOI)}

doi: 10.5474/snapsee-WP3-AT

\section{Citation}

Diese Publikation soll im Literaturverzeichnis wie folgt zitiert werden: Dolinar U., Softic M. and Z. Kozinc (2014), Handbook on Consulting Stakeholders when Applying Best Practices in Sustainable Aggregates Planning, SNAP-SEE, 2014, doi: 10.5474/snapsee-WP3-AT 


\section{Vorwort}

$1 \quad$ Einleitung

1.1 Ziel des Handbuches

1.2 Struktur des Handbuches

1.3 Aggregates Planning as Part of the Policy Cycle

2.1 Gründe zur Teilnahme

2.2 Vorteile

3

$$
\text { Prozessplanung }
$$

3.1 Planungsschritte

3.2 Planungszweck

3.3 Zeitplanung 18

$4 \quad$ Wer sind die Stakeholder und wie involviert man sie $\quad 20$

4.1 Identifizierung der Stakeholder in der Baurohstoffplanung 21

4.2 Gruppierung der Stakeholder gemäß Aufgaben 21

$5 \quad$ Planung einer Veranstaltung mit Stakeholdern $\quad 24$

5.1 Zeitplanung 25

$\begin{array}{lll}5.2 & \text { Struktur } & 27\end{array}$

5.3 Detaillierter Plan 28

$6 \quad$ Workshop Techniken 29

6.1 Gruppeneinteilung 30

6.2 World Café 30

$7 \quad$ Implementierung der Veranstaltung 31

$8 \quad$ Nach der Veranstaltung - Follow-up 33

8.1 Was muss an die Veranstaltungsteilnehmer gesendet werden? 34

$9 \quad$ Empfehlungen 36

9.1 Planung des participativen Prozesses mit den Stakeholdern 37 
9.2 Wer sind die Stakeholder und wie können wir sie einbeziehen 


\section{Vorwort}

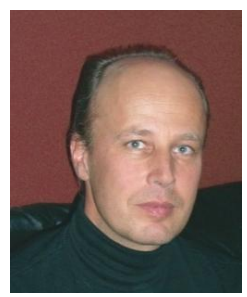

Die Länder in Südosteuropa (SEE) sind reich an Baurohstoffe, also Schotter, Kies, Sand und anderen Rohstoffen die in der Bauindustrie verwendet werden. Allerdings sind diese Mittel sind nicht gleichmäßig verteilt. Weiterhin wird der Zugriff auf diese Ressourcen immer schwieriger aufgrund Infrastrukturentwicklung und Umweltbelastungen. Als Baurohstoffe sind sie zu massiv, ist der Transport zu teuer, daher ist der Zugang zu lokalen Ressourcen entscheidend. In der SEE-Region wird wahrscheinlich die Nachfrage von Baurohstoffen bis 2020 um 50\% ansteigen, um den wachsenden Infrastrukturbedarf umfassend Rechnung zu tragen.

Daher ist ein nachhaltiges Gesamtressourcenmanagement (SARM) und ein nachhaltiges Versorgung Mix (SSM) von Baurohstoffen erforderlich, um die Ressourceneffizienz zu verbessern und die nachhaltige Entwicklung. Die Umsetzung der SARM- und SSM-Konzepte erfordert eine Unterstützung der Rohstoffplanungspolitik. Aufgrund der regionalen Unterschiede und historische Entwicklung, unterscheiden sich die Ansätze der Baurohstoffpolitik, der Planung und des Management innerhalb und zwischen den SEE-Länder. Herausforderungen beziehen sich auf Maßnahmen und Pläne, welche die Baurohstoffe Verwaltung beeinflussen, die unter vielen verschiedenen Rechtsdokumenten verteilt sind, so dass die Koordination und ein umfassendes Verständnis schwierig ist. Es gibt auch einen Mangel der Koordination bei der Planung für die Versorgung aus primären und sekundären Baurohstoffe. Viele SEELänder haben nicht genügend Daten für die Baurohstoffe-Planung, eine unzureichende Kapazität und Kompetenz für die Adressierung entweder primären und / oder sekundären Baurohstoffe Planung, und eine unzureichende Beteiligung der Betroffenen an der Entwicklung der Baurohstoffe-Pläne. Das SNAP-SEE-Projekt hat diese Defizite angesprochen, zur Förderung eines Wachstum einer lebendigen, verantwortlichen und nachhaltigen Baurohstoff -Industrie in der Region.

Der spezifische Zweck von SNAP in Südosteuropa (SEE-SNAP) war die Erstellung und Verbreitung einer Toolbox für Baurohstoff-Planung für Regierungen und Interessengruppen, um ihre Baurohstoff-Planung und Managementprozesse zu verbessern. Das SNAP-SEE Projekt baut auf den Ergebnissen des Projekts "Nachhaltige Baurohstoffe Resource Management" (Sarma) auf, ein vorhergehendes Projekt, gefördertert vom Transnational Cooperation (http://www.sarma.eu). SNAP-SEE wurde ebenfalls von der EU in Südosteuropa finanziert (SEE) Programm Transnationale Zusammenarbeit (SNAP-SEE, SEE / D / 0167 / 2.4 / X) und hatte 27 Partner aus 12 SEE-Ländern und der Türkei. Die Montanuniversitaet Leoben, Österreich, war der federführende Partner. SNAP-SEE war ein 2-Jahres-Projekt, das im November 2014 endete. 
Die SNAP-SEE-Toolbox für Baurohstoffe Planning umfasst 4 Produkte, die miteinander verbunden sind und sich gegenseitig stützen.

1. Eine Vision von Best Practices für Baurohstoff-Planung in Südosteuropa (Vision of Best Practices for Aggregates Planning in South East Europe) "Best Practices" bedeuten eine Vision für einen Übergang zur integrierten, umfassenden nachhaltigen Planung in den SEE-Ländern. Es enthält Diskussionen über die Themen, die angesprochen werden, die in Richtung nachhaltige Planung führen, sowie zur Überprüfung der Komponenten eines nachhaltigen Plan.

2. Wie man nachhaltigen Baurohstoff-Plan erstellt Das 'Wie' Dokument stellt einen Fahrplan für die Planung, einschließlich Diskussionen über den Planungsprozess selbst und den verschiedenen Schritten. Beispiele für Planungsmodule sind vorgesehen, die die Prinzipien, Ansätze und notwendige Aktionen verkörpern, um die Ziele der Visionen zu erreichen.

3. Konsultation der Betroffenen, bei Anwendung der besten Praktiken der Nachhaltigen Baurohstoff-Planung (Consulting Stakeholders when Applying Best Practices in Sustainable Aggregates Planning)

Das "Konsultation" Dokument bietet eine Schritt-für-Schritt-Anleitung für die Planung und Durchführung von Stakeholder-Konsultationen, um zu gewährleisten, dass die Industrie, Behörden, Nicht-Regierungs-Organisationen und der Zivilgesellschaft einen Input bringen.

\section{Daten und Analysen zur Unterstützung von Best Practices in nachhaltigen} Baurohstoffe Planung (Data and Analysis in Support of Best Practices in Sustainable Aggregates Planning)

Dieses Dokument beschreibt die verschiedenen Arten von Daten, die wesentlichen Grundinformationen für den Planungsprozess. Methoden zur Validierung und Daten-Analyse werden bereitgestellt, darunter Ansätze zur Vorhersage der Nachfrage.

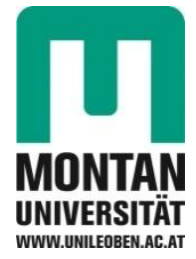

Project Coordinator

Guenter Tiess

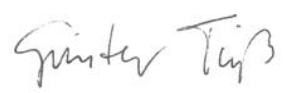

Montanuniversität Leoben

(University of Leoben) 


\section{Einleitung}

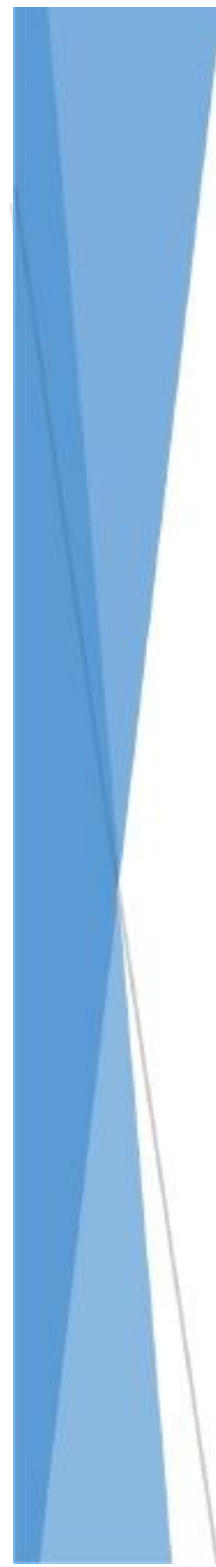

Planung Baurohstoff Versorgung ist eine Aufgabe der nationalen, regionalen und / oder lokalen Regierungen als Teil der Mineralien Planung, Bergbau und Umweltpolitik. Um eine effiziente Planung zu gewährleisten ist es wichtig, mit einer Reihe von Zielgruppen, die in der Baurohstoff Politik und Industrie beteiligt sind oder betroffen zu konsultieren, dh die Beteiligten, einschließlich der Regierungsbehörden und Dienstleistungen auf allen Ebenen, Vertreter der Industrie, der Wissenschaft und Experten, und nicht-staatlichen Organisationen und der Zivilgesellschaft.

Wenn die Akteure in Entscheidungsprozesse, sind verschiedene Ansätze möglich, aus informiert zu gemeinsamen Entscheidungsfindung. Die Auswahl des Ansatzes hängt von den Zielen des Verfahrens und Prozesshaltung .

Dieses Handbuch bietet einen Einblick in den Konsultationen mit den Beteiligten als unterstützende Maßnahme bei der Best Practices in Baurohstoff Planung ist auf nationaler, regionaler oder lokaler Ebene umgesetzt. Es zeigt Vorteile einer partizipativen Ansatz und bietet praktische Ratschläge, wie man die Ziele der Beteiligung der Akteure definieren und praktisch zu planen und umzusetzen, die Konsultationsveranstaltungen. 


\subsection{Ziel des Handbuches}

Baurohstoff Planung bezieht sich auf eine Reihe von öffentlichen Maßnahmen sollen gewährleisten sichere Versorgung der Baurohstoffe für alles, was benötigt, die in einem Land oder einer Region gebaut wird, vom Gebäude-Infrastruktur. Primäre Baurohstoffe sind vor allem Sand, Kies, Schotter; sekundäre Baurohstoffe können Bau- und Abbruchabfälle, hergestellt Baurohstoff, Aushub von Bauarbeiten usw. zurückgeführt werden

Daher ist eines der wichtigsten Ziele, um die Kapazität der Planungsbehörden zu erhöhen, um sich dieser Problematik bewusst zu werden und Einbeziehung von Stakeholdern bei Planungs- und Umsetzungsprozesse zu verbessern. Stakeholder sind "Personen oder Organisationen, die von betroffen sein wird, oder ein Programm, Projekt oder eine Aktion zu beeinflussen" (EG Guidance, 2010). Folglich kann die Beteiligung der Interessengruppen eine Reihe von verschiedenen Aktivitäten von verschiedenen Stellen und mit verschiedenen Gruppen oder Einzelpersonen, von Informationen geben oder sammeln Meinungen, gemeinsame Entscheidungsfindung sind.

Der Zweck dieses Handbuches ist es, praktische Ratschläge bieten:

$\checkmark \quad$ bei der Planung wie man die Kapazität der Baurohstoff Zielgruppen erhöht;

$\checkmark \quad$ Identifizierung verschiedener Gruppen von Akteuren und geeignete Techniken um sie zu involvieren;

$\checkmark$ wie man einen Konsultationsprozess plant;

$\checkmark$ wie man einen Konsultationsprozess und Veranstaltungen durchzuführen hat; und

$\checkmark$ welche Methoden und Techniken bei Konsultationen und Aufbau von Kapazitäten verwendet werden können.

Genauere Erläuterungen zur Baurohstoff Planung finden sich im ersten Produkt der Toolbox für Baurohstoff Planung, eine Vision von Best Practices für Baurohstoff Planung in Südosteuropa. Es enthält allgemeine Informationen über Mineral Planung und Dokumente und präsentiert den Inhalt der Planungsunterlagen und Methoden. 
Primäre Zielgruppe dieses Handbuchs sind Politiker und Beamten der nationalen, regionalen und lokalen Behörden / Regierungen und Einrichtungen aus allen Bereichen der Baurohstoff Planung, einschließlich Bergbau, Bau, Wirtschaft, Umwelt, Abfallwirtschaft, Raumplanung, Wasser, Verkehr usw.

Sekundäre Zielgruppen sind alle Interessengruppen die beteiligt sind oder von der Baurohstoff Planung und Management-Aktivitäten betroffen sind, von Experten, Vertretern der Industrie, Gemeinschaften und Nichtregierungsorganisationen (NGOs).

\subsection{Struktur des Handbuches}

Das Handbuch stellt eine Mischung aus theoretischen Grundlagen und praktischen Tipps basierend auf den Erfahrungen des SNAP-SEE-Projekts. Kapitel 2 erklärt wichtige Vorteile und Prinzipien der Partizipation und dem Engagement der verschiedenen Zielgruppen bei der Planung. Die Kapitel 3-8 sind sehr praxisorientiert und bieten Beratung, wenn jemand vor der Herausforderung steht, die Organisation eines Konsultationsprozesses, bestehend aus einer Reihe von verschiedenen Aktivitäten und Veranstaltungen, durchzuführen. Verschiedene Tipps sind enthalten und Muster-Tabellen werden für die Planung einer Veranstaltung gezeigt. Generische Vorlagen sind zum Download auf http://snapsee.eu/ erhältlich. Das Ziel der Leitlinien und der Vorlagen ist es, Sie durch den Prozess der Teilnahmeplanung zu leiten und hilft Ihnen die verschiedenen relevanten Aspekte zu berücksichtigen. Empfehlungen werden in Kapitel 9 zusammengefasst Abbildung 1.1 zeigt die Struktur des Handbuchs, dh die wichtigsten Schritte bei der Planung der Einbindung von Stakeholdern. 


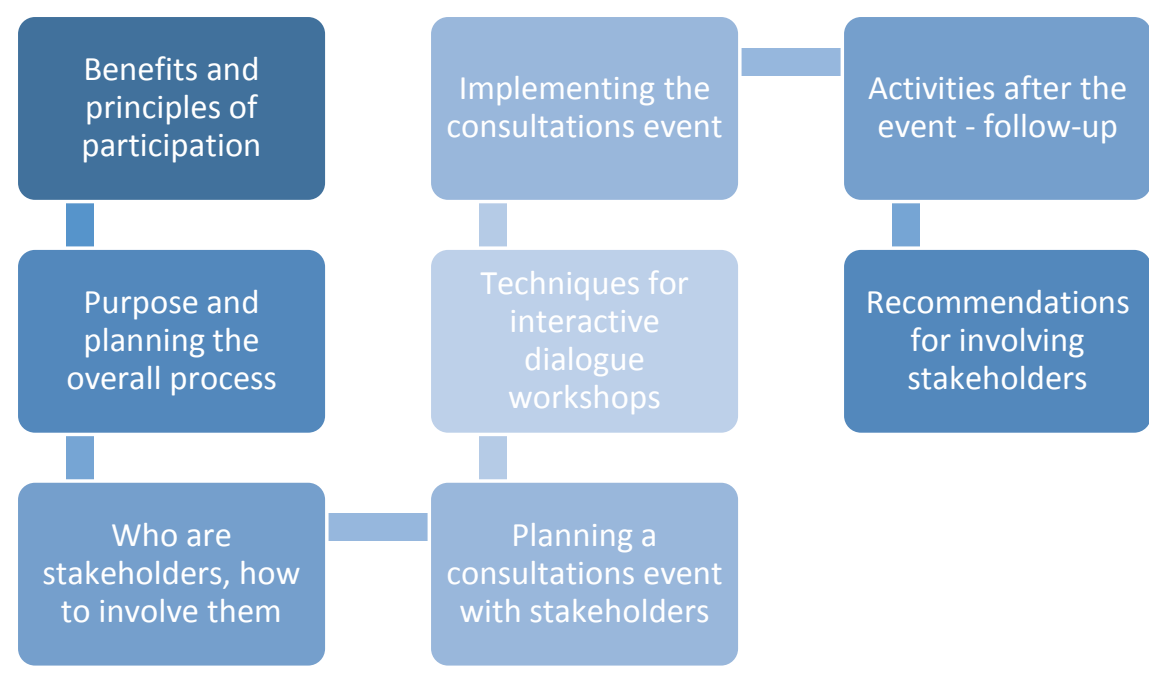

Figure 1.1: Key steps in planning the involvement of stakeholders

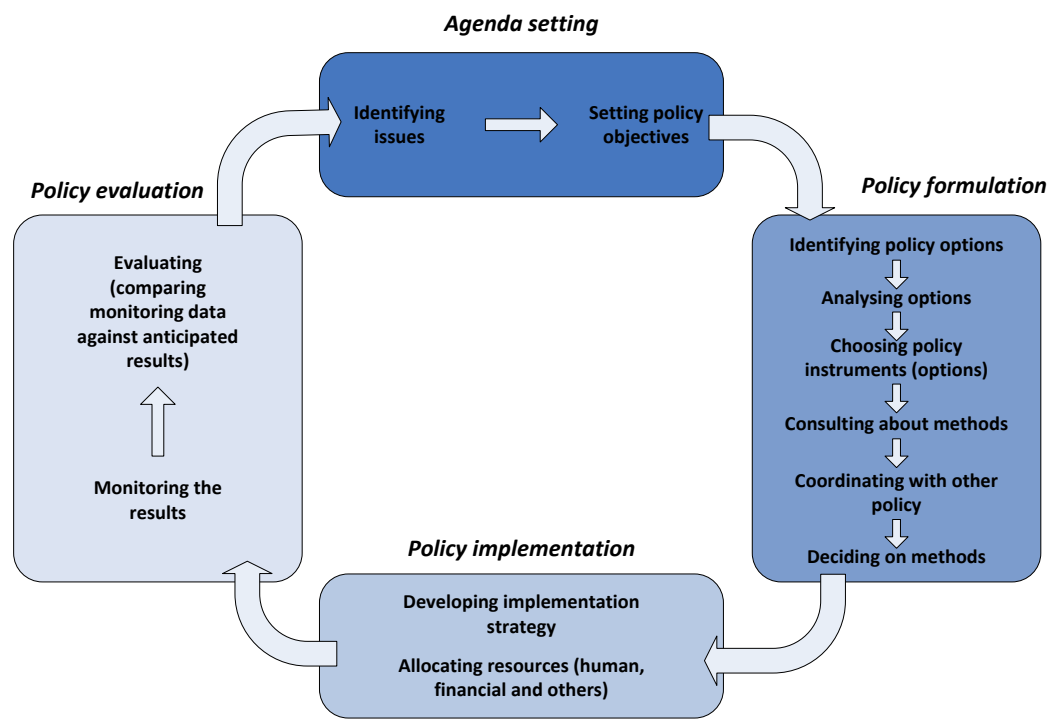

Figure 1.2: Public policy cycle

(http://www.mfe.govt.nz/publications/rma/drafting-issues-jul03/html/page2.html) 


\section{Grundlagen der Stakeholder Involvement}

Im Vergleich zu dem traditionellen öffentlichen Konsultationsansatz, wenn die Entscheidung von einer kleinen Gruppe von Menschen getroffen und später der Öffentlichkeit erklärt wird, hat ein integrativer partizipativer Ansatz mehrere Vorteile. Es resultiert in gut informierten Entscheidungen, die leichter umgesetzt werden können. Echte Probleme werden angesprochen und das Eigentum an Lösungen wird von allen Beteiligten gewährleistet. Schließlich werden die Kosten reduziert und dauerhafte Veränderung zu erwarten.

Dieses Kapitel stellt den Unterschied zwischen der traditionellen öffentlichen Konsultation und dem partizipativen Ansatz der Einbeziehung der Stakeholder dar. Neben den Vorteilen, werden auch verschiedene Möglichkeiten der Einbeziehung von Interessengruppen und Schlüsselprinzipien ihrer Beteiligung erklärt.

Beratungen umgesetzt im SNAP-SEE-Projekt haben eine Reihe von Vorteilen bei der Einbeziehung verschiedener Stakeholder in den Planungsprozess partizipativ gezeigt: unterschiedliche Meinungen wurden geteilt, bessere Lösungen wurden gefunden und die Zusammenarbeit zwischen einer Reihe von Institutionen wurde verbessert. 


\subsection{Gründe zur Teilnahme}

Wenn Fragen wichtig für die Öffentlichkeit diskutiert werden, wie diejenigen, die die nächsten 20 Jahre der Baurohstoff Planung und Bau und mehreren verwandten Branchenbetreffen, sind in der Regel alle einig, dass die Öffentlichkeit in die Diskussion einbezogen werden sollte. Allerdings gibt es einen großen Unterschied zwischen traditionellen öffentlichen Konsultation und einem Stakeholder-Dialog.

Traditionelle öffentliche Konsultation: typischerweise eine kleine Gruppe von Menschen entscheidet, was passieren soll und informiert dann die Betroffenen. Viel Zeit wird zur Erläuterung und Begründung der Entscheidung verbracht. Breiteren Interessengruppen haben begrenzte Möglichkeit zu beeinflussen, was geplant ist und werden in der Regel nur angehört, wenn die meisten Entscheidungen getroffen und niedergeschrieben wurden. Die Umsetzung kann sehr schwierig sein, vor allem, wenn einige Interessen oder Sektoren fühlen, dass ihre Ansichten nicht berücksichtigt wurden (Pound, 2008). Der Prozess dauert länger und hat geringere Möglichkeiten eines gutee Ergebnisses.

Die Behörden sind verpflichtet, bei der Vorbereitung oder Durchführung bestimmter Pläne und Programme bezogen auf die Umwelt, die Beteiligung der Öffentlichkeit bei der Entscheidungsfindung zu respektieren, gemäß dem Übereinkommen von Århus, und der Public Participation Directive (2003/35/EC).

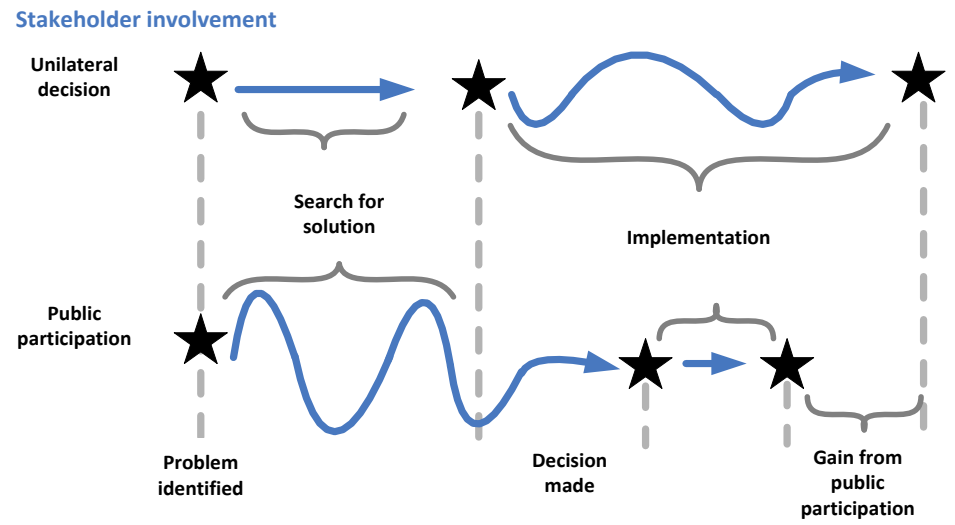

Figure 2.1: Difference between the traditional consultation and stakeholder involvement (adapted after Creighton, 2005) 


\subsection{Vorteile}

Die wichtigsten Vorteile eines gut geplanten und durchgeführten Stakeholder involvement Verfahrens sind:

\section{Vorteile der Einbeziehung von Interessengruppen - Erfahrung des SNAP-SEE- Projekts:}

$\checkmark \quad$ Interessengruppen welche sehr unterschiedliche Organisationen repräsentieren, kamen zusammen und begannen, miteinander zu sprechen, in einigen Fällen zum ersten Mal.

$\checkmark \quad$ erhebliches Interesse an der Teilnahme an Konsultationen und Engagement bei Veranstaltungen wurde beobachtet, auch wenn eine andere Reaktion erwartet worden war.

$\checkmark$ Die Zusammenarbeit wurde zwischen den Behörden der verschiedenen Sektoren, wie zB Bergbau, Umwelt, Wasser, Landnutzungsplanung, Transport etc. verbessert auch auf unterschiedlichen Ebenen (national, regional, lokal).

$\checkmark$ Bewusstsein über die Bedeutung der Planung wurde erhöht.

$\checkmark$ Die Notwendigkeit der Vorbereitung einer nationalen Rohstoffpolitik wurde erkannt.

$\checkmark \quad$ Haupthindernisse oder Probleme bei der Planung wurden identifiziert (zB Vorgangsweisen, illegale Gewinnung, mangelndes Bewusstsein etc.).

$\checkmark \quad$ Vorschläge für Lösungen wurden vorgeschlagen und waren für die Mehrzahl der beteiligten Gruppen akzeptabel.

$\checkmark \quad$ Bedeutung von Recycling von Baurohstoffen wurde gefördert.

$\checkmark$ Eine Grundlage für die künftige Zusammenarbeit wurde erstellt oder verbessert. 


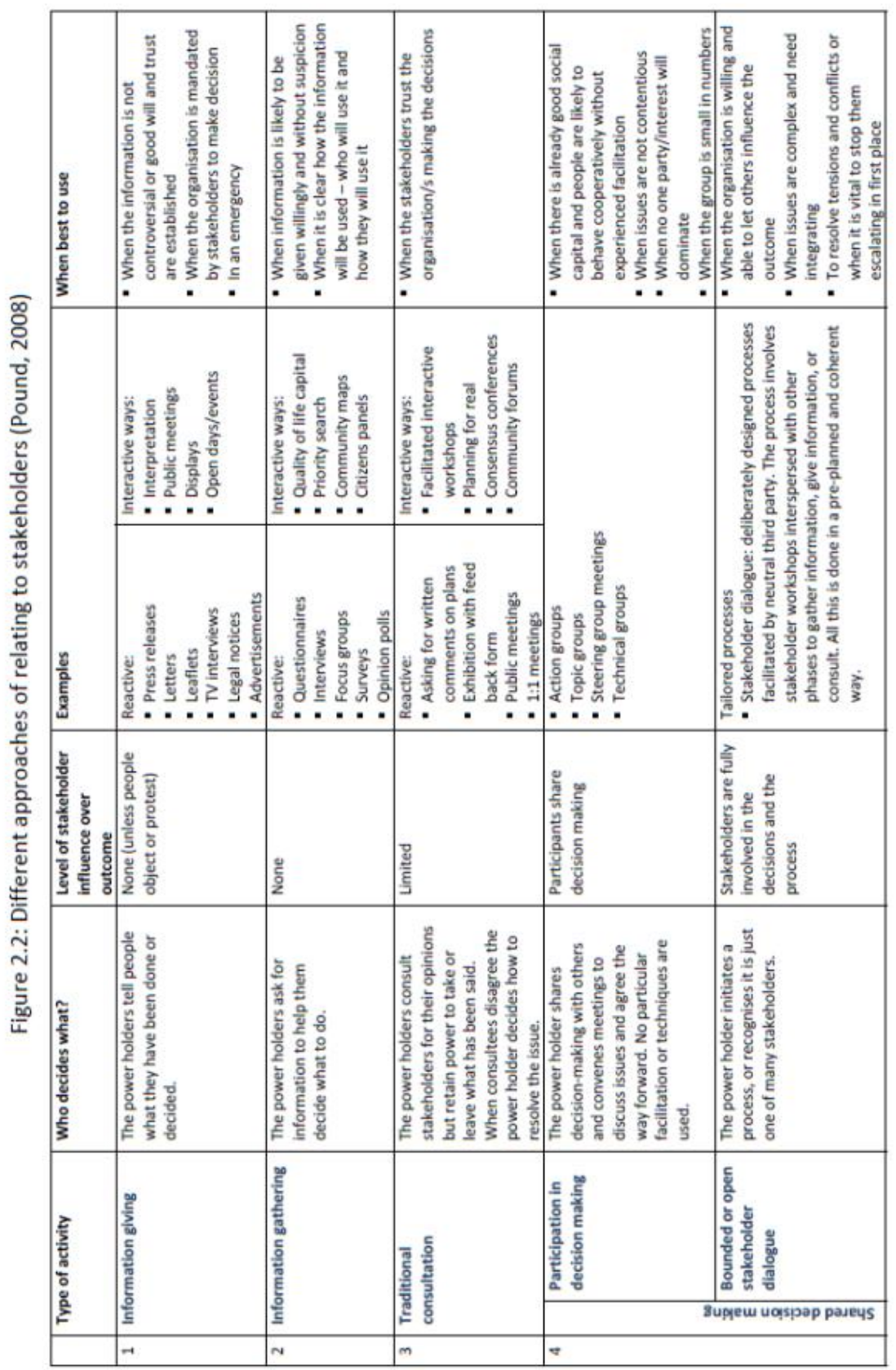




\section{Prozessplanung}

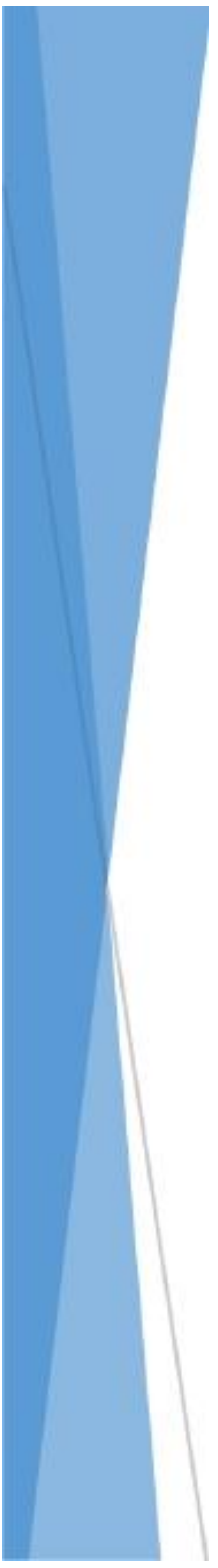

Wenn wir uns auf die Verbesserung einer Rohstoff/Baurohstoff Planungspolitik durch ehrliche Einbeziehung der Interessengruppen entscheiden, sollten wir den Prozess ihrer Teilnahme, Ziele eines solchen Prozesses, welche Aktivitäten wir unternehmen und die Rollen der einbezogenen Personen, sorgfältig planen und definieren.

Dieses Kapitel konzentriert sich auf die Planung des partizipativen Prozesses, während die nächsten Kapitel die Analyse der Akteure erklären und Tipps für die Planung und Umsetzung der Veranstaltungen in dem Prozess bereitstellen.

Im SNAP-SEE-Projekt war es das übergeordnete Ziel des Konsultationsprozesses mehr nachhaltige Versorgung von Baurohstoffen in den teilnehmenden Ländern oder Regionen zu erreichen. Die Ergebnisse für jedes Land / Region waren die sektorübergreifende Analyse des aktuellen Ansatzes für Baurohstoffplanung und nationale / regionale Leitlinien zur Verbesserung der Baurohstoffplanung. Die erzielte Ergebnis enthalten: besseres Verständnis der Bauroh-stoffplanung, Einigung über akzeptable Lösungen, erhöhte Kapazität von SARM und SSMKonzepten und bewährte Verfahren in anderen Ländern, gesteigertes Bewusstsein für nachhaltige Konzepte und bessere Kenntnis der aktuellen Daten und Methoden. 


\subsection{Planungsschritte}

Sobald wir uns entschieden haben, Interessengruppen in einem partizipativen Prozess für eine nachhaltige Baurohstoffplanung zu involvieren, ist es entscheidend, mit sorgfältiger Planung und Vorbereitung zu starten. Planung des partizipativen Prozesses beinhaltet mehrere Schritte, ob wir einen einjährigen Prozess oder ein einzelnes Ereignis planen. Diese und die folgenden Kapitel erklären fünf Teile der effektiven Planung von einem partizipativen Prozess:

1. Das Verständnis der Situation - Zweck und Ergebnisse

2. Prozessplanung - Aktionsplan

3. Veranstaltungsdesign: Planung, Durchführung und Nachbereitung

4. Aufgabe oder Sitzungsplanung

5. Praktische Organisation.

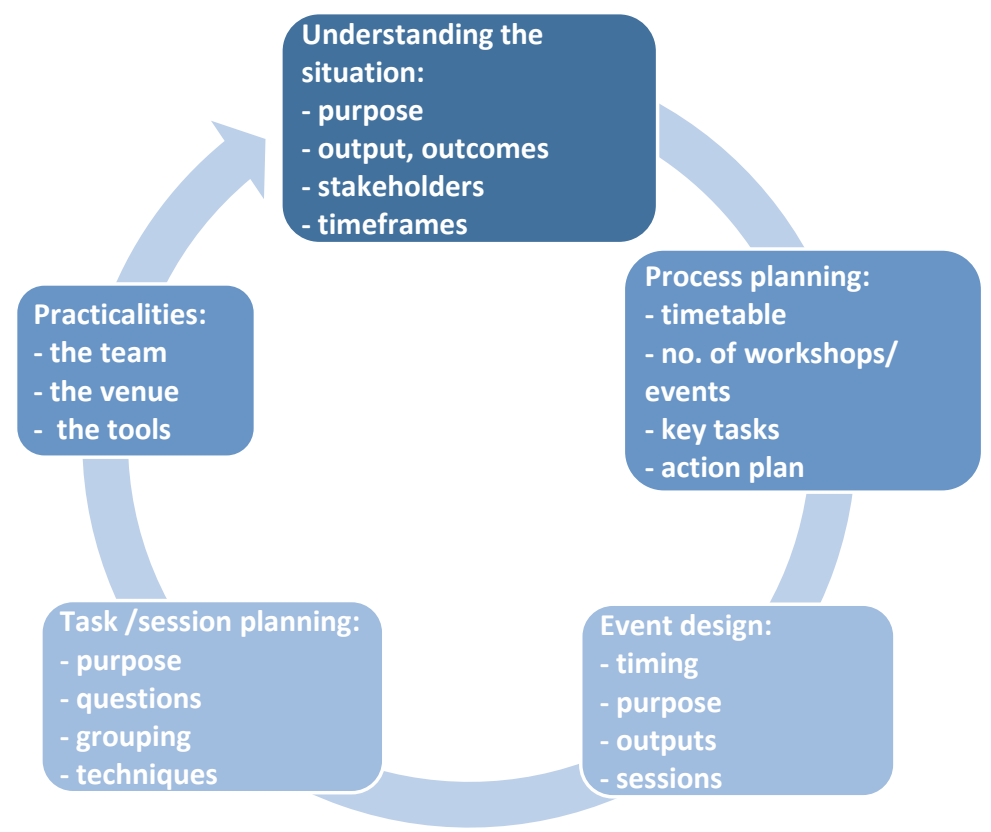

Figure 3.1: Steps for effective planning of a participatory process (Pound, 2008) 


\subsection{Planungszweck}

\begin{tabular}{|l|l|}
\hline Defining the purpose of the process - leading questions (Pound, 2008) \\
\hline Zweck & $\begin{array}{l}\text { Was ist der Hauptzweck dieses Verfahrens / Veranstaltung? } \\
\text { Was ist der Hauptzweck dieses Verfahrens / Veranstaltung? } \\
\text { Was ist realistisch und erreichbar innerhalb der zur Verfügung stehenden } \\
\text { Ressourcen, Personal, Zeit und Budget? } \\
\text { Was meinen die Initiatoren / Problem Halter zu erhoffen, bis Ende des Pro- } \\
\text { zesses, was sie jetzt noch nicht haben? } \\
\text { Wie werden sie wissen, dass der Prozess erfolgreich war? Wie wird es sich } \\
\text { anfühlen, aussehen? }\end{array}$ \\
\hline Ergebnisse & $\begin{array}{l}\text { Was sind die festen Ergebnisse? } \\
\text { Beispiele: Spezialberichte, Aktionsplan, Prioritätenliste }\end{array}$ \\
\hline Wirkung & $\begin{array}{l}\text { Was wollen die Menschen als Ergebnis dieses Prozesses ändern? } \\
\text { Relationale Ergebnissen beinhalten: bessere Beziehungen, besseres Ver- } \\
\text { ständnis, mehr Käufe und Eigentum, aktive Unterstützung, stärkere Teams, } \\
\text { mehr Vertrauen, verstärkte Zusammenarbeit, Verringerung der Spannun- } \\
\text { gen, bessere Entscheidungen } \\
\text { Inhaltsergebnisse können sein: besser verwaltetes Umwelt, nachhaltigere } \\
\text { Nutzung / Entwicklung, Einigung über optimale Vorgangsweisen }\end{array}$ \\
\hline Personen & $\begin{array}{l}\text { Für wen ist dieser Prozess? } \\
\text { Wer sollte involviert werden und in welcher Befugnis? } \\
\text { Wer sind die Stakeholder? (siehe auch Kapitel 4)? }\end{array}$ \\
\hline Inhalt & $\begin{array}{l}\text { Was ist der Schwerpunkt der Diskussion? } \\
\text { Was sins die Belange, Themen und Anliegen? }\end{array}$ \\
\hline
\end{tabular}

Zweck des Beteiligungsprozesses - Beispiele aus den SNAP-SEE Projekt Beratungen:

$\checkmark \quad$ Einbeziehung der bedeutendsten Stakeholder in die Baurohstoffplanung, wie nationale Regierungen, lokale Behörden, den privaten Sektor, die bürgerliche Gesellschaft und Gemeinden.

$\checkmark \quad$ Einigung über Baurohstoff-Nutzungsplans, der die nachhaltige Entwicklung garantiert und erhöhen der Möglichkeiten zur Wiederverwertung.

$\checkmark$ Aufnahme der sekundären Baurohstoffe in die Baurohstoffplanung als mögliche Quelle für Entwicklung einer Infrastruktur in einer umweltfreundlichen Art.

$\checkmark \quad$ Um in Einklang mit den strategischen Richtlinien des Landes und der EU zu sein.

$\checkmark \quad$ Um Synergien zwischen den verschiedenen Pläne und Strategien über Baurohstoffe zu erzielen. 


\subsection{Zeitplanung}

Die Zeitplan Aufstellung enthält Zeitskala in der oberen Reihe (Monate ab Beginn der Planung bis wir den Prozess abschließen möchten) und verschiedene Arten von Aktivitäten / Einschränkungen auf der linken Seite. In die Tabelle fügen wir ein:i

$\checkmark$ Projekt / andere wichtige Meilensteine, die unsere Prozess beeinflussen;

$\checkmark$ Beschränkungen, wie Urlaub, Ferien, Schulferien, andere wichtige Ereignisse etc .;

$\checkmark$ Daten geeignet für geplante Veranstaltungen im Prozess (Workshops mit Stake-holdern, kleinere Meetings etc.);

$\checkmark$ Aktivitäten des Koordinationsteam; und

$\checkmark$ Aktivitäten mit Stakeholdern. 


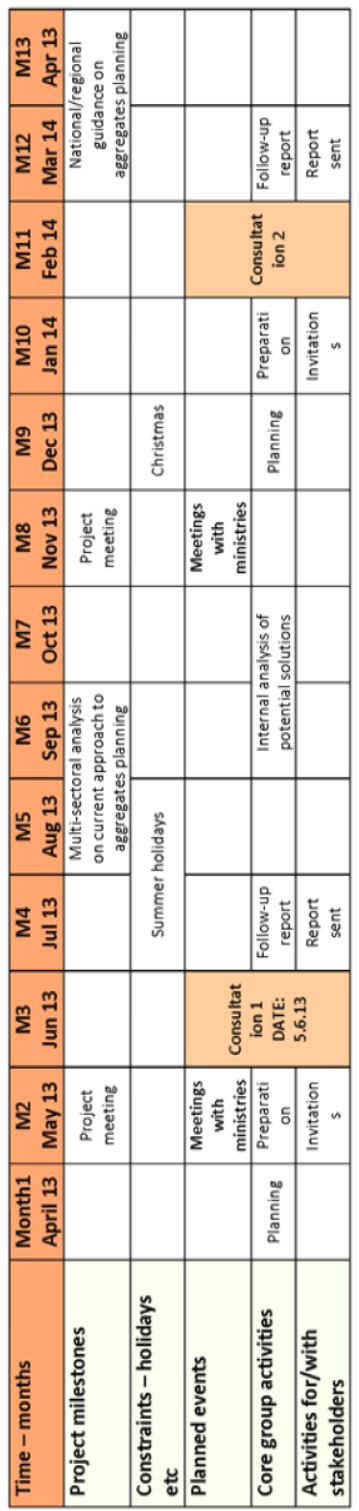




\section{Wer sind die Stakeholder und wie involviert man sie}

Interessengruppen können als eine Person, Gruppe oder Organisation definiert werden, welche wahrscheinlich betroffen sind oder ein Interesse in den zu treffenden Entscheidungen haben.

Dies umfasst alle Organisationen oder Personen, die verantwortlich für Baurohstoffplanung sind, direct durch die Planungsvorgänge betroffen sind oder involviert bzw. betroffen sind durch Steinbrucharbeiten sowie Vor- oder Nachaktivitäten der Steinrbucharbeiten. 


\subsection{Identifizierung der Stakeholder in der Baurohstoffplanung}

Table 4.1: Stakeholders gemäß Arten von Organistationen

Regierung / Behörden aus allern oben auf-
geführten Bereichen:
- Nationale Regierung: Ministerien,
- Rgenturen, öffentliche Institute
- Logionalregierung
- le / lokale Entwicklungsagenturen
- Aufsichtsämter
- Gremien auf EU Ebene

Experten:

- Geologische Dienste, Forschungsinstitute und ähnliches

- Institute aus anderen oben genannten Sektoren (Raumplanung, Umwelt, Naturschutz, Abfallwirtschaft, Verkehr, Wasserwirtschaft, Landwirtschaft, Forstwirtschaft usw.)

- Universitäten und andere Bildungsorganisationen

- Bergbau Experten, Geologen usw.

- Internationale Institute

\section{Gesteinsindustrie:}

- Quarry Besitzer

- Quarry Betreiber

- Hersteller von rezyklierten Gesteinskörnungen

- Arbeiter und Arbeitervereine

- Verbände - regionale, nationale und internationale

- Handelskammern

- Bauindustrie

- Baurohstoff Verbraucher

- Gewerkschaften

Gemeinschaften und NGOs, Freiwilligensektor:

- Grundbesitzer

- Lokale Einwohner, Bauern

- Nicht-Regierungsorganisationen in den Bereichen Umweltschutz, Ökologen, Vogelschutz etc.

- Bürgervereinigungen

Medien, Allgemeinheit

\subsection{Gruppierung der Stakeholder gemäßAufgaben}

\begin{tabular}{|l|l|}
\hline Gruppe & Aufgabe \\
\hline Koordination & $\begin{array}{l}\text { Vertreter der koordinierenden Organisation(en), d.h. Projektpartner und } \\
\text { Beobachter aus jedem Land / Region: kooperieren in allen Projektaktivitä- } \\
\text { ten, definieren, planen und setzen den Beteiligungsprozess um, organisie- } \\
\text { ren Veranstaltungen, schreiben Texte und Berichte. Sie leiten und realisie- } \\
\text { ren den gesamten Prozeßes und bereiten Dokumente vor. }\end{array}$ \\
\hline Arbeitsteam & $\begin{array}{l}\text { Vertreter von Schlüsselorganisationen verantwortlich für Baurohstoffpla- } \\
\text { nung für primäre und sekundäre Baurohstoffe: sie bereiten Beiträge zur } \\
\text { Erstellung von Analysen und Berichten, und nehmen an den Veranstaltun- } \\
\text { gen mit Stakeholdern teil. Sie haben eine aktive Rolle während des gesam- }\end{array}$ \\
\hline
\end{tabular}




\begin{tabular}{|l|l|}
\hline & $\begin{array}{l}\text { ten Prozesses. Neben den Veranstaltungen können kleinere Treffen mit } \\
\text { ihnen organisiert werden, um die ordnungsgemäße Planung, Durchführung } \\
\text { und Folgevorgänge des Teilnehmerprozesses sicherzustellen udn aktuelle } \\
\text { Daten für die Berichte zu garantieren. Sie liefern Beiträge zu den Doku- } \\
\text { menten and nehmen an den Veranstaltungen der Stakeholder teil. }\end{array}$ \\
\hline $\begin{array}{l}\text { Vertreter der } \\
\text { führenden } \\
\text { Gruppen der } \\
\text { Stakeholder }\end{array}$ & $\begin{array}{l}\text { Vertreter der oben aufgeführten verschiedenen Gruppen von Akteuren } \\
\text { (Sektoren und Arten von Organisationen), die auch wichtige Entschei- } \\
\text { dungsträger und Meinungsmacher repräsentieren. Sie sind durch die Akti- } \\
\text { vitäten der Baurohstoffplanung betroffen und ihre Stimme muss gehört } \\
\text { werden. Ihre entscheidende Rolle ist es, ihre Meinungen und Informatio- } \\
\text { nen beiden Konsultationens-Veranstaltungen vorzulegen. Bei Bedarf kön- } \\
\text { nen sie auch dazwischen konsultiert werden. Sie nehmen an den Verans- } \\
\text { taltungen teil. }\end{array}$ \\
\hline $\begin{array}{l}\text { Allgemeinheit, } \\
\text { Media }\end{array}$ & $\begin{array}{l}\text { Vertreter der Medien und der Öffentlichkeit, die an dem Thema interes- } \\
\text { siert sind: sie werden durch die allgemeinen Werbeaktivitäten, wie die } \\
\text { Projekt-Website, Artikel, Broschüren und Prospektrichtet angesprochen. } \\
\text { Sie werden über das Projekt informiert. }\end{array}$ \\
\hline
\end{tabular}

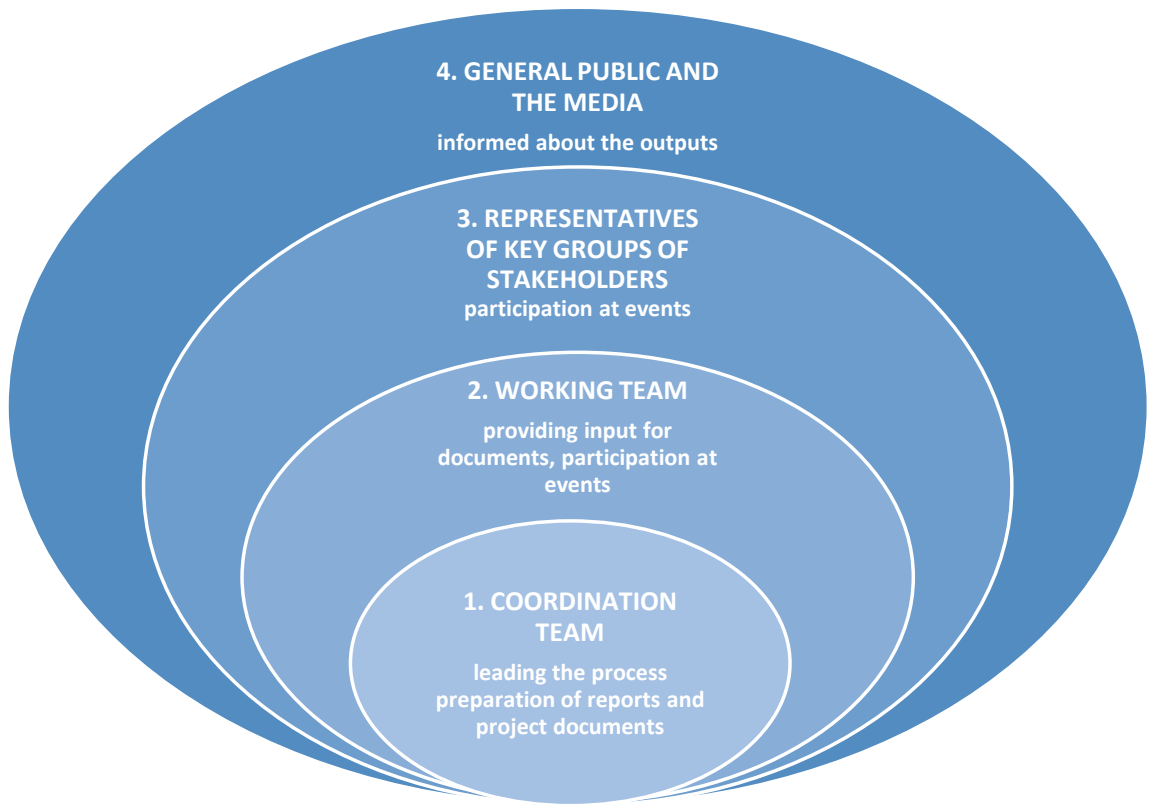

Figure 4.1: Einteilung der Stakeholder in 4 Gruppen gemäß des Grades ihrer Mitwirkung 
Erfahrung bei Involvierung unterschiedlicher Stakeholder im SNAP-SEE Projekt :

$\checkmark \quad$ Die Interessen der verschiedenen Zielgruppen sind sehr unterschiedlich. Ihre Meinungen unterscheiden sich sehr und können gegesätzlich sein. Daher müssen Sie sorgfältig überlegen, wie man mit ihnen allen zusammenarbeitenkann und wie man eskalierende Konflikte vermeidet. Einige Tipps zu vermittelnden Techniken sind in Kapitel 6 vorgestellt.

$\checkmark \quad$ Vertreter der verschiedenen Gruppen haben ihr Interesse an einer Teilnahme bei Konsultationen gezeigt.

$\checkmark \quad$ Es ist wichtig, nicht nur die zuständige Organisation, sondern auch die richtige Person, die mit dem Thema befasst ist, zu identifizieren.

$\checkmark \quad$ Es ist sehr schwierig, Vertreter der Behörden zu motivieren. Es ist wichtig, sie über die Vorteile und die zu erwartenden Ergebnisse der Konsultationen zu informieren.

$\checkmark \quad$ Die Industrie ist sehr motiviert, zu kooperieren und wir müssen beachten, dass sie gleichermaßen vertreten sind. Sie sind am meisten interessiert, dass die Behörden stabile und faire Arbeitsbedingungen für ihre Tätigkeiten garantieren.

$\checkmark$ Experten Organisationen und Experten aus verschiedenen Bereichen werden wich-tige Einblicke in verschiedene Themen bieten.

$\checkmark \quad$ Die Vertreter der Umweltbereiche, NGOs und Gemeinden sind auch daran interessiert, in den Dialog von Anfang an eingebunden zu sein, so dass ihre Meinungen be-rücksichtigt werden können. Die Erfahrung zeigt, dass sie nicht von vornherein gegen (Gewinnungs) Operationen sind, aber sie werden auch zu oft erst nach dem letzten Abschnitt des Verfahrens informiert. 


\section{Planung einer Veranstaltung mit Stakeholdern}

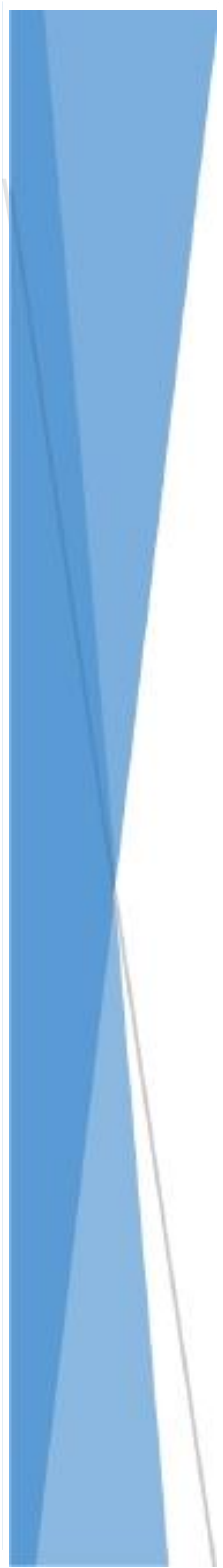

Im SNAP-SEE-Projekt wurden 20 Konsultationsveranstaltungen in 10 Ländern organisiert, mit über 800 Teilnehmern von 90 Organisationen, repräsentierend alle Zielgruppen. Dieses Kapitel fasst die Erfahrungen der Organisation solcher Veranstaltungen zusammen, gleichzeitig werden Details in der abschließenden ProjektVeröffentlichung (unter http://snapsee.eu zugänglich) vorgestellt. Es ist entscheidend früh genug mit der Planung zu beginnen, gut vorzubereiten, Aufgaben den Teammitgliedern zuzuweisen, und das Follow-up nach der Veranstaltung sicher zustellen. All dies wird dazu beitragen, das Ziel einer Veranstaltung erfolgreich zu erreichen, dh, gemeinsame Lösungen für bessere Baurohstoffplanung zu finden. 


\subsection{Zeitplanung}

Arbeitsschwerpunkte für die Organisation einer Veranstaltung sind in Abbildung 5.1 zusammengefasst. Sie werden in der zeitlichen Reihenfolge aufgebaut: was muss vor dem Ereignis durchgeführt werden, bei der Veranstaltung und nach dem Ereignis. 


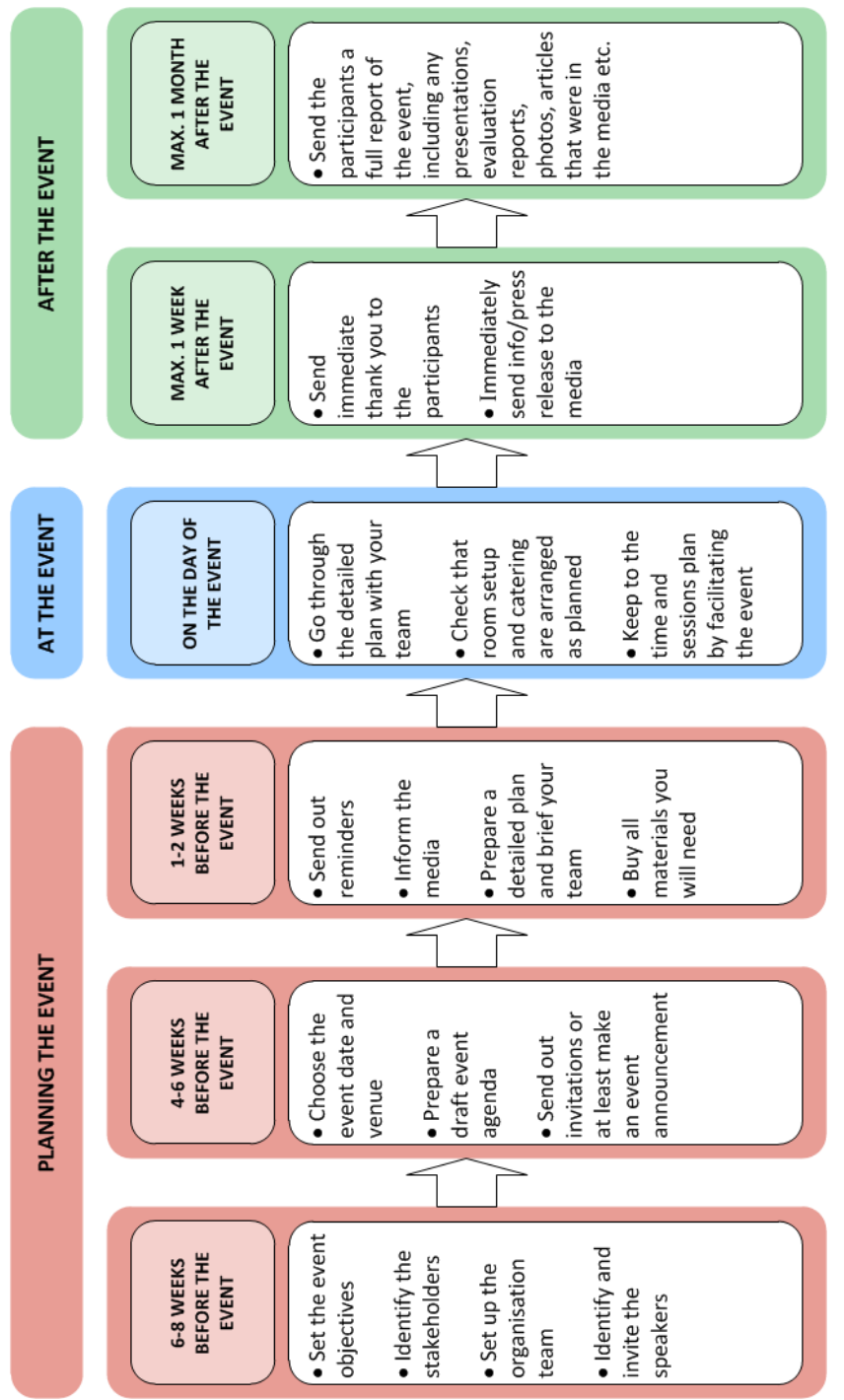

Figure 5.1: Zeitplan für die Organisation einer Stakeholder Veranstaltung (Softić et al., 


\subsection{Struktur}

Liste der verfügbaren Kompetenz-bildenden Präsentation zur Verwendinung in Englisch und in Sprachen von Süd Ost Europa:

1. Sustainable Aggregates Planning in South East Europe (presentation of the SNAP-SEE project and preceding SARMa project)

2. Providing a Sustainable Supply Mix (SSM) of Aggregates

3. Legal and regulatory framework for aggregates supply at the EU level

4. Aggregates: Planning - Demand - Data - Green Public Procurement

5. Recycling of aggregates and related data

6. Aggregate Intelligence System \& Geographic Information System to support resource efficiency; example of a GIS system

7. Material flow accounts

8. Aggregates demand forecasting

9. Decision modelling

10. EU Green Public Procurement (GPP) Policy and Road Criteria

11. Sustainable Aggregates Planning - Life-cycle approach

Bei den SNAP-SEE waren die Themen auf das Gesamtziel gerichtet: was getan werden sollte um die Baurohstoffplanung in unserem Land/unserer Region nachhaltiger zu machen. Das beinhaltet die folgenden Fragen::

$\checkmark \quad$ Was ist der aktuelle Stand der Planung; Was sind Themen, Engpässe, Lücken?

$\checkmark \quad$ Wer ist zuständig für die Baurohstoffplanung? Kooperieren die verschiedenen Behörden miteinander? Wenn ja, wie funktioniert es? Wenn nicht, warum nicht? Wer blockiert die Zusammenarbeit?

$\checkmark$ Wie sind die Verfahren? Sind sie zu langsam? Wenn ja, warum?

$\checkmark$ Was sind die wichtigsten Hindernisse, vom Standpunkt der Industrie?

$\checkmark$ Was sind die wichtigsten Probleme wahrgenommenvon NGOs, Gemeinden und Einwohnern??

$\checkmark \quad$ Was sind die Vorschläge, um diese Hindernisse zu überwinden?

$\checkmark \quad$ Welche Fälle aus anderen Ländern können als Vorbild verwendet werden (in geeigneter Weise auf die Situation des anderen Landes / der Region angepasst), um die Probleme lösen?

$\checkmark$ Welche Lösungen sollten umgesetzt werden, unter welchen Bedingungen, von wem, in welchem Zeitrahmen? 


\subsection{Detaillierter Plan}

\section{Planung der Konsultationsveranstaltungen - Erfahrung des SNAP-SEE-Projekts:}

$\checkmark$ Wir müssen die Planung rechtzeitig im Vorhinein beginnen (mindestens 3 Monate), um interessante Vortragende zu gewinnen und Teilnehmer zu motivieren.

$\checkmark$ Wir müssen darauf achten, nicht die Veranstaltung nicht zu nahe bei Feiertage zu organiseren, da Menschen dazu neigen, einige Urlaubstage anzuhängen.

$\checkmark$ Auf Einladung müssen wir eindeutig den Zweck der Veranstaltung angeben und dass Antworten der Teilnehmer zu ausgewählten Themen gesammelt werden.

$\checkmark$ Es sollte eine geeignete Anzahl von Teilnehmern sein, um Arbeit in Gruppen (nicht zu klein und nicht zu viele, zwischen 20 und 60) zu ermöglichen.

$\checkmark \quad$ Wir sollten mit der Anzahl der Präsentationen nicht übertreiben, da Teilnehmer müde werden können und eventuell früher gehen.

$\checkmark$ Wir müssen sehr sorgfältig zu definieren, was wir gerne in der Diskussion oder in Gruppenübungen heraus finden möchten. Ein kompetenter und gut vorbereiteter Moderator (und / oder Unterstützer) hat eine entscheidende Rolle bei einem erfolgreichen Ergebniss der Diskussion.

$\checkmark$ Kaffeepausen und Mittagspause sind eine wichtige Gelegenheit für die Teilnehmer, die verschiedene Zielgruppen repräsentieren, um die Gespräche fortzusetzen und sich informell auszutauschen.. 


\section{Workshop Techniken}

Die Diskussion in kleineren Gruppen hat zahlreiche Vorteile. In diesem Kapitel sind einige Techniken die für interaktiven Workshops verwendet werden vorgestellt. Es gibt in der Tat kein Rezept, wie man die richtigen Techniken für einen der Workshops auswählt; dies ist der kreative Teil des gesamten Prozesses, wo wir mit unseren Ideen spielen, um eine Reihe von Maßnahmen zu setzen, die uns zu unserm, für diese Veranstaltung, gesetztem Ziel, führen.

Der Schlüssel ist, die am besten geeigneten Techniken zu finden, um unser Ziel in der zur Verfügung stehenden Zeit für Einzelsitzungen, zu erreichen,

Bei den SNAP-SEE Veranstaltungen hat die Erfahrung gezeigt, dass Diskussionen in kleinen Gruppen von 7-10 Personen mehrere Vorteile hat, zB mehr Menschen beteiligen sich an der Diskussion, gibt es mehr Austausch von Meinungen und Erfahrungen, und verschiedene Lösungen werden identifiziert und verbessert. 


\subsection{Gruppeneinteilung}

Einige Möglichkeiten, um Menschen zu gruppieren:

$\checkmark$ "Durchnummerieren" der Anwesenden und einteilen aller "Einser" in einen Arbeitstbereich Eins, alle "Zweier" zum Arbeitsbereich Zwei usw. Menschen aus den gleichen Organisationen sitzen oder stehen in der Regel zusammen, auf diese Art und Weise werden Sie sie mischen

$\checkmark$ Teilen Sie den Menschen eine Farbe oder Form zu, während sie sich registrieren und teilen sie diebGruppen anhand der gleichen Farben oder Formen ein. Dies kann durch Aufkleben eines farbigen Punkt auf den Namensschildern oder indem sie innen Namensschilder in verschiedenen Farben geben, geschehen. Dann sind alle Teilnehmern mit z.B. orange Farbe zu bitten, um eine Gruppe zu gehen, alle z.B. "Roten" zu einer anderen Gruppe und so weiter.

\subsection{World Café}

Eine der Sorten des Karussells Brainstorming ist die so genannte World CaféMethode. Dies ist eine einfache Methode der Förderung der Konversation unter den Teilnehmern zu definierten Fragen. Das Verfahren ermöglicht das Sammeln von Ideen und Erfahrungen der einzelnen Gruppenmitglieder in einem kurzen Zeitraum (1-2 Stunden). Hauptmerkmale dieses Verfahrens sind:

$\checkmark \quad$ Ein Tisch für eine Frage, die Teilnehmer sitzen um den Tisch herum..

$\checkmark \quad$ Legen sie auf die Tische Flip Charts und Marker (auf jedem Tisch eine andere Farbe)

$\checkmark \quad$ Wir stellen Snacks, Kekse und Kaffe auf den Tisch um es ähnlich eineem Kaffehaustisch zu machen, diese Methode ahmt eine entspannnte Diskussion in einem Kaffehaus nach.

$\checkmark \quad$ Ein Teilnehmer jeder Gruppe wird als "Gastgeber" (oder Moderator) nominiert und wird an diesem Tisch während des ganzen Prozesses bleiben.

$\checkmark \quad$ Nach 20 minütiger Diskussion wechselt die Gruppe den Tisch.

$\checkmark$ Der Gastgeber präsentiert kurz die Ergebnisse der vorherigen Gruppe und dann kann die Gruppe ihre eigenen Antworten bearbeiten.

$\checkmark \quad$ Sie können wählen, dieselbe Frage an alle Gruppen zu stellen, in diesem Fall bleiben die Teilnehmer immer am selben Tisch und wechseln die Tische nicht. 


\section{Implementierung der Veranstaltung}

Arbeitsschwerpunkte für den Tag, an dem das Ereignis durchgeführt wird, sind Anweisung des Teams, die Einrichtung der Zimmer und praktischen Einteilungen, gute Moderation und Abschluss der Veranstaltung, so dass die Teilnehmer mit einem guten Eindruck abschließen.

Am Tag der Veranstaltung, müssen wir sicher sein, dass der detaillierte Veranstaltungsplan, den wir vorbereitet haben, funktionieren und der Tag reibungslos ablaufen wird. Dazu gehört die Überprüfung des Veranstaltungsplans, Einsatzbesprechung des Teams und die Sicherstellung, dass alle praktischen Vorkehrungen bei der Hand sind.

Eine der wichtigsten Erfahrungen der SNAP-SEE Veranstaltungen sind, dass man flexibel sein muss - denn sicher wird etwas nicht genau nach Plan gehen, aber man sollte ruhig bleiben und sich an die neuen Umstände anpassen und das Ergebnis wird doch noch erreicht werden. 
- sich selbst vorstellen

- entspannend sein

- zuversichtlich sein

- den entsprechenden Stimmklang verwenden und Fragen stellen

- jeden ermutigen, teilzunehmen

- angemessen reagieren: durch Bestätigung, Aufmunterung, Verständnis zeigen

- Stille akzeptieren, damit arbeiten, nicht zu versuchen diese zu füllen

- die Ansichte eines jeden gleichberechtigt behandeln

- mit schwierigen Personen auskommen

- alles mitschreiben, über was gesprochen wurde
- übertreiben bei der Zusammenfassung

- unterbrechen

- vorzeitig zusammenfassen

- eigene Meinungen und Ansichten aufnötigen

- jemanden zu einem "Sonderfall" machen

- Geduld verlieren 


\section{Nach der Veranstaltung - Follow-up}

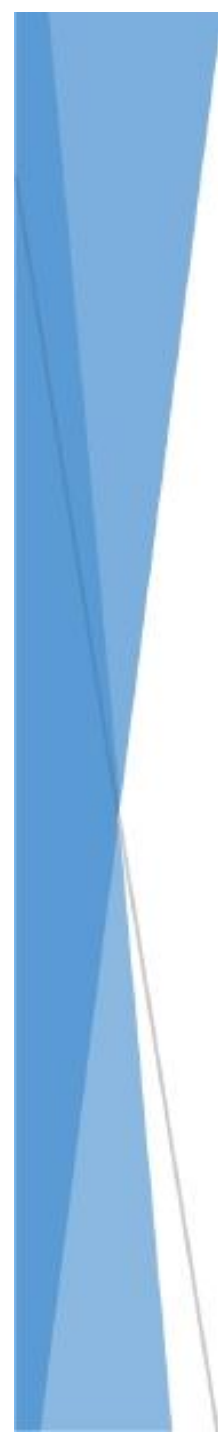

Egal wie erfolgreich die Veranstaltung war und wie zufrieden die Teilnehmer waren,

kann das Ergebnis sehr schnell verloren gehen wenn Sie geeignete Folgemaßnahmen zur Veranstaltung nicht sicher stellen.

Es ist daher wichtig, dass Sie die vereinbarten Termine für den Versand von Material und Informationen einhalten. Sie sollten mit den Teilnehmern in 1-2 Wochen nach der Veranstaltung kommunizieren.

In diesem Kapitel wird kurz zusammengefasst, was Sie den Teilnehmern senden sollen, warum und wie man sich für den nächsten Vorgang oder nächsten Schritt vorbereitet.

Nach den SNAP-SEE Veranstaltungen erhielten die Teilnehmer einiges Informationsmaterial ts per E-Mail: Kurzbericht über die Veranstaltung, die Aufnahme des (interaktiven) Diskussion, Teilnehmerliste und die Präsentationen. 


\subsection{Was muss an die Veranstaltungsteilnehmer gesendet werden?}

Sie sollten an die Teilnehmer senden:

$\checkmark \quad$ Ein kurzer Bericht von der Veranstaltung mit Abschriften von Wort-fürWort-Aufnahmen aus den Werkstätten - nicht versuchen, zusammenzufassen, aber lassen Sie es in den Worten der Teilnehmer geschrieben.

$\checkmark \quad$ Erklären Sie, wie Sie die Ergebnisse des Workshops und ihre Beiträge nutzen werden.

$\checkmark \quad$ Schließen Sie auch die Bewertungsergebnisse ein.

$\checkmark \quad$ Hängen Sie die gegebenen Präsentationen an, zusammen mit einer gescannten anwesenheitsliste und Fotos.

$\checkmark$ informieren Sie über die nächsten Schritte oder Veranstaltungen.

\section{Nach der Veranstaltung:}

$\checkmark$ senden Sie einen Bericht über die Veranstaltung / Workshop an alle Teilnehmer (mit Präsentationen und Fotos) und an diejenigen, die eingeladen wurden, aber nicht teilnehmen konnten..

$\checkmark$ Bereiten Sie eine Pressemitteilung über die Veranstaltung für die Medien vor.

$\checkmark$ Halten Sie die Teilnehmer auf dem Laufenden und in beteiligen Sie sie auch bei Themenverwandten Veranstaltungen.

Wenn das Ereignis abgeschlossen ist, sollten Sie auch in Ihrer Organisation und dem Koordinationsteam bewerten, und setzen Sie es in die Perspektive des Gesamtprozesses:

$\checkmark$ Haben wir das Ziel der Veranstaltung erreicht?

$\checkmark$ Wer waren die Teilnehmer? Haben sie die (Haupt-) Stakeholder Organisationen repräsentiert?

$\checkmark \quad$ Wer hat gefehlt? Warum? Was können wir tun um sie für den Vorgang zu gewinnen?

$\checkmark$ Was werden wir mit dem Feedback tun?

$\checkmark$ Wer wird den Projektbericht vorbereiten?

$\checkmark \quad$ Brauchen wir zusätzliche Analysen, Informationen usw.? Müssen wir zusätzliche Experten konsultieren um das herauszufinden? 
Wenn wir anfangen, über die nächste Veranstaltung nachzudenken, sollten wir alle Schritte überdenken und abschätzen, wie Aktivitäten (falls nötig) das nächste Mal verbessert werden können. 


\section{Empfehlungen}

Als Zusammenfassung werden Empfehlungen für jedes Kapitel zusammengefasst. Sie bieten die wichtigsten Richtlinien, die bei der Planung eines partizipativen Prozesses, der Implementierung eines einzelnen Workshops oder Einsatz einer der interaktiven Techniken berücksichtigt werden müssen.

Die Empfehlungen basieren auf den Erfahrungen der SNAP-SEE Projektpartner bei den Konsultationen, die sie gehalten haben, der Handbuch Autoren und der Literaturübersicht. Wir empfehlen dringend, die vorgeschlagenen Methoden auszuprobieren, ihre Vorteile herauszufinden und während des Vorgangs zu lernen. 
Der Zweck des SNAP-SEE Projektes war es, Kapazitäten der Akteure zu erhöhen und Werkzeuge zu entwickeln zur Sicherstellung einer nachhaltigen Versorgung mit Baurohstoffen in der Süd-Ost-Europa. 27 Partner aus 13 Ländern haben ihren aktuellen Stand der Baurohstoffplanung analysiert, Leitlinien zur Verbesserung des Prozesses entwickelt - und sich über die Vision von Best Practices für Baurohstoff Planung in Südosteuropa geeinigt.

Eines der Ergebnisse ist, dass jedes Land den Baurohstoff oder Rohstoff Plan für die nächsten 20 Jahre entwickeln sollte. Allerdings wird die Planung durch die Regierungen getan und kann nicht allein von den Projektpartnern durchgeführt werden. Die Tool-box für Aggregate Planning ist ein Ergebnis der Projekterfahrung und präsentiert erhärtende Belege für jedes interessierte Unternehmen, das vor solchen Herausforderungen steht. Es ist wichtig, die Akteure partizipativ so früh wie möglich in den Prozess einzubeziehen. An dieser Stelle sind die wichtigsten Empfehlungen zusammengefasst.

\subsection{Planung des participativen Prozesses mit den Stakeholdern}

$\checkmark \quad$ Wir müssen klar den Zweck der Konsultation definieren, was wir erreichen wollen, wer teilnehmen sollte und wir wir die gesammelten Informationen verwenden wollen.

$\checkmark \quad$ Die Teilnehmer müssen über den Zweck, ihre Rolle und wie sie die Entscheidungen beeinflussen können informiert werden.

$\checkmark \quad$ Das übergeordnete Ziel des Prozesses ist es, mehr nachhaltige Versorgung von Baurohstoffen durch Einbeziehung aller betroffenen Zielgruppen zu erreichen.

$\checkmark$ Das Ergebnis kann sein: ein besseres Verständnis eines Baurohstoff Planungsprozesses, Einigung über Lösungen, erhöhte Kapazität bei SARM und SSM-Konzepten und bewährte Verfahrensweisen in anderen Ländern, erhöhtes Bewusstsein für nachhaltige Ansätze und gesteigertes Wissen der aktuelle Daten und Methoden.

$\checkmark \quad$ Der Zeitplan des Prozesses und einzelne Schritte müssen sorgfältig definiert werden, um das Gesamtziel zu erreichen. 


\subsection{Wer sind die stakeholder und wie können wir sie einbeziehen}

$\checkmark$ Jeder Konsultationsprozess hat unterschiedliche Zielgruppen. Wir müssen sorgfältig festzulegen, wer die Gruppen verantwortlich für Entscheidungen sind, welche beteiligt oder beeinflusst sind.

$\checkmark \quad$ wichtigen Zielgruppen in der Baurohstoff Planung sind: Regierungsbehörden der verschiedenen Sektoren, Baurohstoffindustrie, Experten, lokale Gemeinschaften und NGOs und die Öffentlichkeit und die Medien.

$\checkmark$ Bedürfnisse der verschiedenen Stakeholder-Gruppen sind unterschiedlich, und wir müssen dies bei der Planung von Art und Umfang der Beteiligung berücksichtigen. Einige werden von Ihnen werden gerne an eintägigen Workshops teilnehmen, während bei anderen müssen wir ein kurzes Treffen organisieren, um relevante Informationen von ihnen zu erhalten.

$\checkmark \quad$ Die Interessen der verschiedenen Zielgruppen variieren sehr. Ihre Meinungen unterscheiden sich extrem und können im Gegensatz zu einander sein. Deshalb müssen wir sorgfältig abwägen, wie man mit allen von ihnen zusammenarbeiten und eskalierende Konflikte vermeiden kann.

$\checkmark \quad$ Die Industrie ist sehr motiviert, zu kooperieren und wir müssen aufpassen, dass sie gleichermaßen vertreten sind. Sie sind daran interessiert, dass die Behörden stabile und faire Arbeitsbedingungen für ihre Operationen gewährleisten.

$\checkmark$ Expertenorganisationen und Experten aus verschiedenen Bereichen werden wichtige Einblicke in verschiedene Themen bieten.

$\checkmark$ Die Vertreter der Umweltbereiche, NGOs und Gemeinden sind auch daran interessiert, in den Dialog von Anfang an eingebunden zu sein, so dass ihre Meinungen berücksichtigt werden kann. Die Erfahrung zeigt, dass sie nicht von vorne herein gegen (Gewinnungs-) Tätigkeiten sind, aber sie werden zu oft bis zur letzten Stufe ignoriert. 


\section{Quellen und weiterführende Literatur}

Århus Convention: UNECE Convention on Access to Information, Public Participation in Decision-making and Access to Justice in Environmental Matters. http://www.unece.org/env/pp/treatytext.html

British Geological Survey (2006). Planning4Minerals: A Guide on Aggregates. http://www.bgs.ac.uk/planning4minerals/assets/downloads/86210 P4M A Gui de On Aggregates.pdf

Chalkiopoulou, F. \& Hatzilazaridou, K. (2011). How to achieve aggregates resource efficiency in local communities, a joint manual, for stakeholders' decision making on the local level, SARMa (SARMa - SEE/A/151/2.4/X).

http://www.sarmaproject.eu

Chreighton, J. L. (2005). The Public Participation Handbook: Making Better Decisions Through Citizen Involvement. John Wiley \& Sons, Inc.

Dolinar, U. \& Kozinc, Z. (2013). Stakeholder involvement as a key factor for sustainable aggregates planning and management, Proceedings, 6th International Conference on Sustainable Development in the Minerals Industry (SDIMI), Milos, Greece.

Erricsson, M. \& Särkkä, P (2013). Socio-economic impact of the Finnish extractive industries. Proceedings, 1st International Conference on Sustainable Development in the Minerals Industry (SDIMI 2003), Milos, Greece, pp. 243-251.

Getzner, M. et al. (2010). People, parks and money. Stakeholder involvement and regional development: a manual for protected areas. Klagenfurt, Verlag Johannes Heyn.

Global Reporting Initiative (2002). Sustainability Reporting Guidelines.

International Council on Mining and Metals (2003). Community Development Toolkit. ICMM, London.

Korff, von Y. et al. (2010). Designing Participation Processes for Water Management and Beyond. Ecology and Society, vol. 15., No. 3.

http://www.ecologyandsociety.org/vol15/iss3/art1/

Marega, M. (2011). Guidelines on Stakeholder Engagement in Preparation of Integrated Management Plans for Protected Areas. NATREG project.

http://www.zrsvn.si/dokumenti/64/2/2010/Guidelines on stakeholder engage ment REC 1978.pdf 
MMSD (2002). Breaking new ground. Final report on the Mining, Minerals and Sustainable Development Project. IIED, London.

http://pubs.iied.org/pdfs/9084IIED.pdf

New Zealand Planning Institute (2013). Quality planning New Zealand.

http://www.qualityplanning.org.nz/index.php

Nielsen, K. (2003). Sustainable development indicators in the aggregate industry. A Norwegian perspective. Proceedings, 1st International Conference on Sustainable Development in the Minerals Industry (SDIMI 2003), Milos, Greece, pp. 285289.

Participation compass (2013). participationcompass.org

Participation Directive: Directive 2003/35/EC of the European Parliament and of the Council of 26 May 2003 providing for public participation in respect of the drawing up of certain plans and programmes relating to the environment and amending with regard to public participation and access to justice Council Directives 85/337/EEC and 96/61/EC.

Pound, D. (2008). Stakeholder dialogue, A good practice approach to participation. Dialogue Matters Ltd.

Softić, M., et al. (2011). Training Manual for Organisation of National Seminars within the DMCSEE project. DMCSEE (DMCSEE - SEE/A/091/2.2/X).

Šolar, V. S., Shields, D. J.,\& Zelič, U. (2012). Sustainable Aggregates Resource Management: experience learnt and shared within South East Europe. Ljubljana: Mining and Metallurgy Quarterly (RMZ - Rudarsko metalurški zbornik).

Šolar, S. \& Shields, D. (2011). The SARMa Project: enhancing sustainable aggregates resource management and supply in Southeast Europe. In: Martens, P. (ed.) Proceedings, $5^{\text {th }}$ Conference on the Sustainable Development in the Mineral Industry (SDIMI 2011). June 14-17, Aachen, DE. Essen, DE: RWTH Aachen University.

The Environment Council (2004). Good Practice for Stakeholder Engagement in the Aggregates Sector. http://www.sustainableaggregates.com/library/docs/ samp/l0138 samp 1 019.pdf

Tiess, G. (2001). General and International Mineral Policy. Focus Europe, Springer.

Tiess, G. \& Chalkiopoulou, F. (2011). SARM and SSM at the Regional, National and Transnational Level manual, SARMa (SARMa - SEE/A/151/2.4/X).

Van der Veen, P. \& Strongman, J. (2003). Sustainable development - The way forward for the mining industry, Proceedings, 1st Int/nal Conference on Sustainable Development in the Minerals Industry (SDIMI 2003), Milos, Greece, pp. 17-23. 


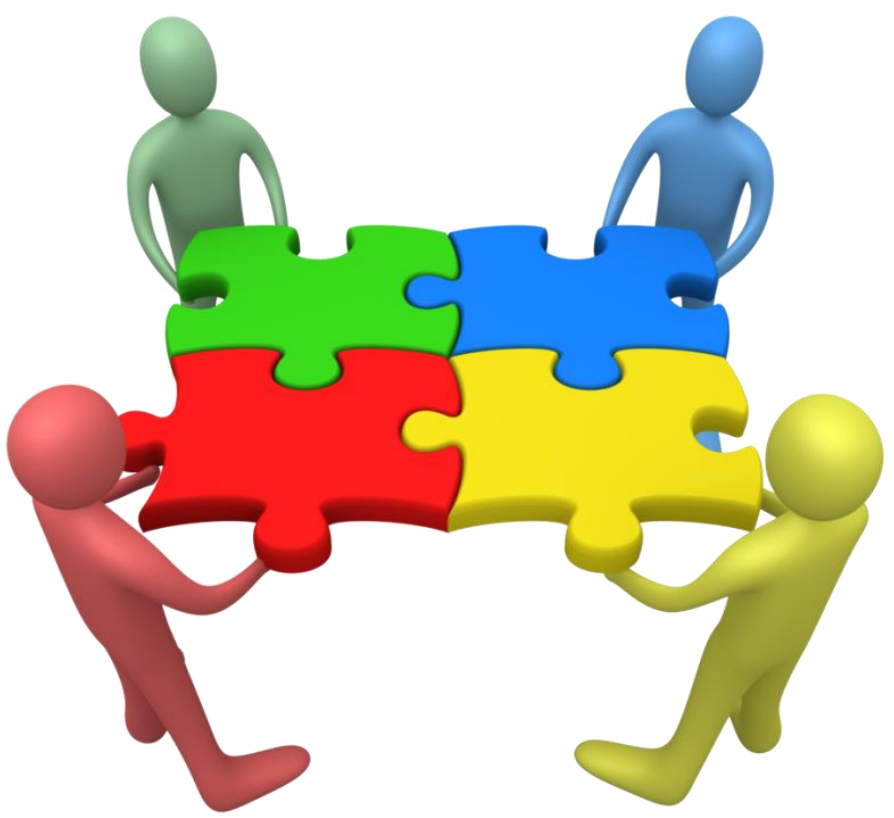

Baurohstoff-Planung in Süd-Ost Europa (SNAP-SEE) 